\title{
OPTIMAL CONTROL OF TRACTOR-TRAILER SYSTEM IN HEADLANDS
}

\author{
T. Oksanen ${ }^{1}$, A. Visala ${ }^{2}$
}

\begin{abstract}
A tractor-trailer combination or a tractor with a towed implement is a common form for agricultural machines, such as seed drillers, harrows, fertilizers or sprayers. When optimizing field traffic, it is most significant to minimize the time spent in the headlands. Therefore the behavior of tractor-trailer combination in a headland is studied in this paper.

The tractor-trailer combination is modelled as a differential equation system or dynamic model with six states and two inputs. The movement is restricted into 2D-plane. The supplementary requirements for the model are the ability to reverse driving, allowing the switch-back turning and the standard formulation of optimal control problem. The dynamic model is parametrized in terms of mechanical parameters of the vehicle.

Optimal control theory and tools are reviewed. The minimum-time optimal control problem of headland turning with tractor-trailer vehicle is presented. Vehicle's mechanical and field's geometrical constraints are considered. The optimal control problem is highly nonlinear so it cannot be solved analytically.

Numerical algorithms for solving this kind of optimal control problems demand high computational cost and cannot be solved in real-time even with modern desktop computers. The optimal control problem is solved with fixed vehicle parameters and varying headland parameters, headland angle and width. In order to use optimal control problem solutions in real-time applications, the solutions in variable headland cases are approximated with simple trajectories. The rules for selecting a turning method with fixed vehicle parameters and certain headland parameters are presented.
\end{abstract}

KEYWORDS. Optimal control, Vehicle Modeling, Headlands, Turning.

\section{INTRODUCTION}

It is very possible that there is no driver in tractor in the future, but the tractors are like robots. Robot research has usually separated its methodology fields, like perception, navigation, path planning, positioning, servo control etc. Path planning is one of the most difficult part, the driving reference plan has to be made by robot for every field. Usually the farmer has learned the best way to drive during several years, especially in family farms where farmer really knows his/her fields. Robot can also have memory but for the first time a good reference path has to be generated from a priori information. Path planning has to optimize some criterion, it may contain travelled distance, fuel consumption, trampling of field, quality of task and also time.

When optimizing field traffic, it is most significant to minimize the time spent in the headlands. The less turnings are made in field, the less time the turnings totally take. But also efficient vehicle behaviour in one turning is important.

\footnotetext{
${ }^{1}$ Research Scientist. Automation Technology laboratory, Helsinki University of Technology, Finland.

${ }^{2}$ Professor. Automation Technology laboratory, Helsinki University of Technology, Finland.
} 
Tractor-trailer combination is a common form of agricultural machines. The example of such machine is shown in figure 1, trailer is a towed combined fertilizer-seed drill.

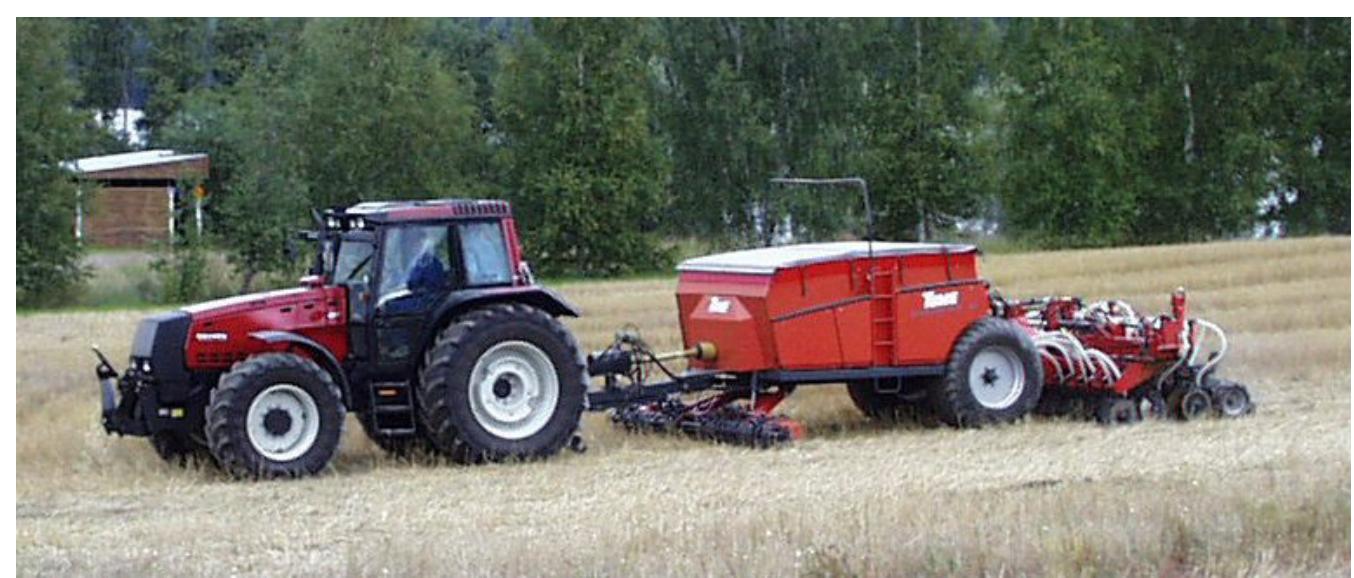

Figure 1. The example of tractor-trailer vehicle.

\section{MODELLING OF THE VEHICLE}

The dynamic model will be used in the optimal control problem so the system should be modelled carefully. The vehicle is considered to move in 2D-plane, thus the tractor itself contains 3 degrees of freedom (DOF), $x_{R}, y_{R}$ and $\theta$. The trailer can be added to tractor model with one DOF, the angle of drawbar $\varphi$. These parameters are visualized in figure 2 .

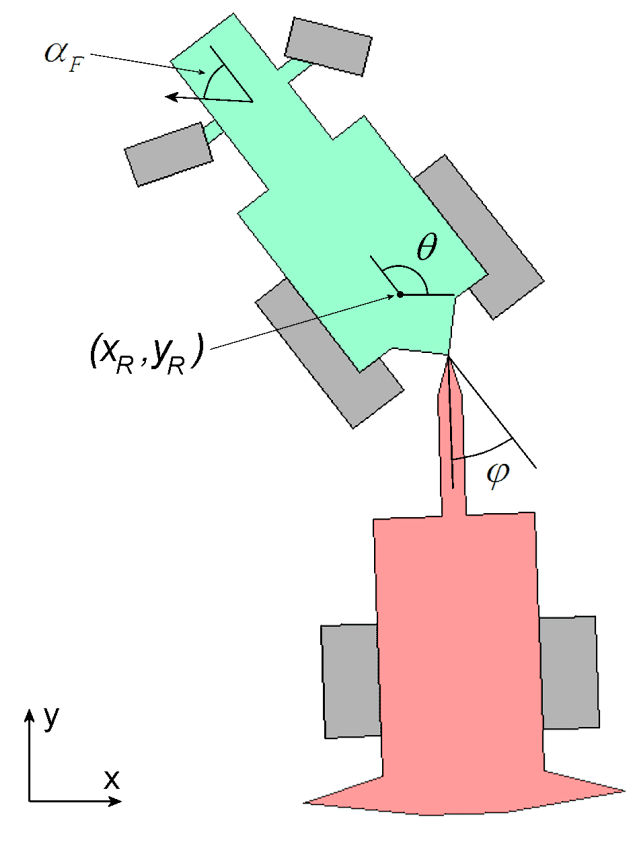

Figure 2. Vehicle parameters

The physical parameters of the vehicle are the axle spacing of the tractor $(a)$, the spacing between tractor rear axle and drawhook (or drawbar coupling) (b) and the spacing between drawhook and trailer axle $(c)$. This parametrization allows the joint of the vehicle to be in the drawhook, in the rear hitch drawbar coupling or in the free joint-drawbar. The functional component of the trailer can be in front of the trailer axle or behind it $(d)$, if it is behind the parameter $d$ is positive and vice versa. Only the front wheels of the tractor are steerable. The parameters $a-d$ are visualized in figure 3 . 


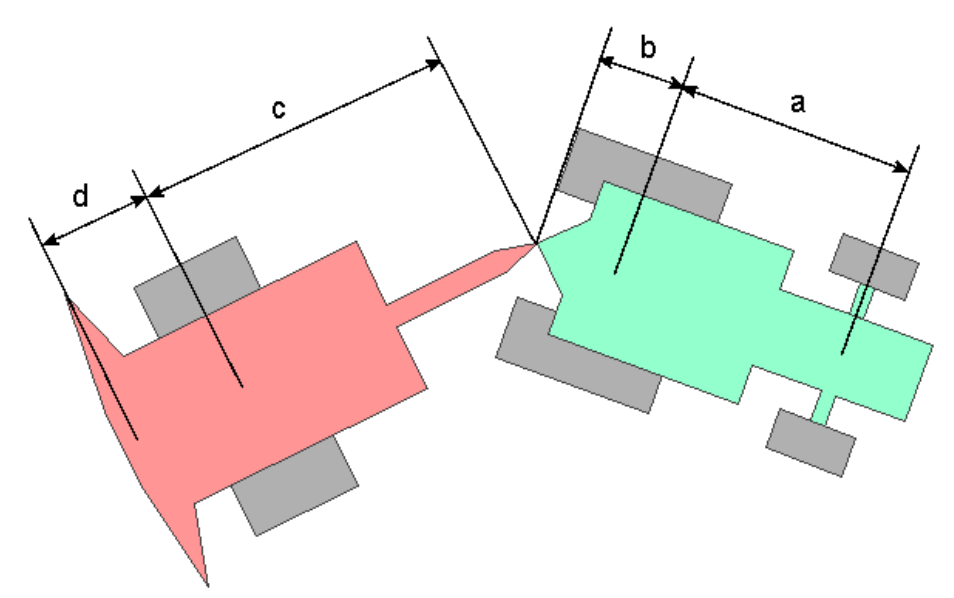

Figure 3. Physical parameters of the model.

The dynamic model is presented in (1) and the auxiliary variables $\left(x_{H}, y_{H}, v_{H}, \beta\right)$ needed are presented in (2). The model contains six states and two controls, $x$ and $u$ respectively. The symbols are $\left(x_{R}, y_{R}\right)$ is the position of tractor rear axle, $\theta$ is steering angle, $\varphi$ is drawbar angle. There are two physical controls for the system, the velocity of the tractor (rear)wheels and the steering angle of the front wheels. In order to avoid the derivation of states in checking of optimal control constraints (e.g. acceleration of vehicle), the physical controls are considered as states $\left(v_{R}, \alpha_{F}\right)$ of the system and the change (time derivative) of those are the inputs of the system $\left(a_{R}, \omega_{F}\right)$.

$$
\begin{aligned}
& \left\{\begin{array}{l}
\dot{x}_{R}=v_{R} \cos (\theta) \\
\dot{y}_{R}=v_{R} \sin (\theta) \\
\dot{\theta}=\frac{1}{a} v_{R} \tan \left(\alpha_{F}\right) \\
\dot{\varphi}=\frac{1}{c} v_{H} \sin (\beta-\varphi-\theta)-\dot{\theta} \\
\dot{v}_{R}=a_{R} \\
\dot{\alpha}_{F}=\omega_{F}
\end{array}\right. \\
& \dot{x}_{H}=\dot{x}+b \sin (\theta) \cdot \dot{\theta} \\
& \dot{y}_{H}=\dot{y}-b \cos (\theta) \cdot \dot{\theta} \\
& v_{H}=\sqrt{\dot{x}_{H}^{2}+\dot{y}_{H}^{2}} \\
& \beta=\arctan \left(\dot{x}_{H}, \dot{y}_{H}\right) \\
& x=\left[\begin{array}{llllll}
x_{R} & y_{R} & \theta & \varphi & v_{R} & \alpha_{F}
\end{array}\right]^{T}, u=\left[\begin{array}{ll}
a_{R} & \omega_{F}
\end{array}\right]^{T}
\end{aligned}
$$

In order to complete the model, the physical limits of the vehicle has to be considered. The dynamic system has been chosen so that all the limits are type of saturation. The limits for the example vehicle are shown in (4). The first one is the limit for velocity (negative speed corresponds to driving backwards), second one is the acceleration and deceleration limit, the third is the maximum angle of front wheel steering angle, the fourth is the angular velocity of steering wheels and the last one is the limit for drawbar angle.

$$
\left\{\begin{array}{l:l}
v_{R}^{\min } \leq v_{R} \leq v_{R}^{\max } & \mathrm{km} / \mathrm{h} \\
a_{R}^{\min } \leq a_{R} \leq a_{R}^{\max } & \mathrm{km} / \mathrm{h} / \mathrm{s} \\
-\alpha_{F}^{\max } \leq \alpha_{F} \leq \alpha_{F}^{\max } & \circ \\
-\omega_{F}^{\max } \leq \omega_{F} \leq \omega_{F}^{\max } & \circ / \mathrm{s} \\
-\varphi^{\max } \leq \varphi \leq \varphi^{\max } & \circ
\end{array}\right.
$$


Usually the numerical optimal control problem solvers can handle one state constraints more efficiently than all together. Therefore it is important to divide simple one state constraints and mixed state constraints. The constraints for the last three states and both controls can be considered as static constants opposite to the limits of three first states, they describe the position of vehicle in field. Therefore those limits for three first states are separated from the others. Generally speaking the limits of first three states cannot be separated, the mixed path-constraints has to be used for them.

\section{Optimal Control Problem}

An optimal control problem (OCP) can be formulated as:

$$
\begin{aligned}
& \min J(u)=\int_{t_{0}}^{t_{f}} F(x(t), u(t), t) d t, \\
& \dot{x}(t)=f(x(t), u(t), t), t_{0} \leq t \leq t_{f}, \\
& C(x(t), u(t), t) \leq 0, t_{0} \leq t \leq t_{f}, \\
& E\left(x\left(t_{0}\right), x\left(t_{f}\right)\right) \leq 0,
\end{aligned}
$$

where $x$ is state vector, $f$ is dynamics function, $t_{0}$ and $t_{f}$ are time limits, $J$ cost function, $C$ path constraint function and $E$ boundary condition function. (von Stryk et al., 1992)

The earliest origins of optimal control are in the calculus of variations in 17 th century. The classic optimal control theory was developed mainly in the 1950's and 1960's, the motivation was space and aeronautics engineering. The principle of optimality, dynamic programming, Bellman equations and Pontryagin maximum principle come from that era. The problem of these theories is that they solve the optimal control problem for certain problem types, like problems with linear dynamics and a quadratic cost function, for which the analytic solution can be obtained from necessary and sufficient conditions of optimality. For nonlinear dynamics and non-quadratic cost function numerical solver is needed. (Bryson 1996).

The numerical methods that rely on solving the necessary conditions of the Pontryagin maximum principle are usually called as indirect methods. The direct numerical methods are recent, have improved during the 1990's and are still being improved. In direct methods the infinite dimensional problem (from maximum principle) is approximated with a finite dimensional problem. The philosophy in direct methods is to discretize the problem in time and to approximate the states and the controls in those time points and convert the optimal control prolem (OCP) to a nonlinear linear programming (NLP) problem. The NLP problem is a standard optimization problem that can be solved efficiently with numerical tools.

One of the first direct methods was called direct shooting through parametrization of controls $u(t)$. The method relies on numerical integration to satisfy the dynamic model. Another method uses discretation of both the controls and the states in time, model dynamics is satisfied in discrete points with equation constraints. End-point constraints are also of equation type, but path constraints are inequality constraints in NLP. States are approximated with polynomials or more advanced approximations between discrete time points. The result is a nonlinear programming problem. This method is often called direct collocation. In the very basic direct collocation method the dimension of the NLP problem grows quite large, so the calculation time of numerical NLP solvers is quite long. The disadvantage of direct collocation methods is that they produce less accurate solutions than indirect methods. The usability of the solution depends on the accuracy requirements of the original optimal control problem. The other disadvantage in this method is that the numerical solution may be a local minimum, not global, and the local minimum found can far from the global. (von Stryk et al., 1992). 
One of the latest direct numerical methods is the so called pseudospectral approximation which is an adaptive algorithm, (Ross et al., 2002). The pseudospectral method can handle efficiently especially discontinuities and other nonsmoothness of state trajectories but has also faster convergence.

There are commercial tools available for solving OCP numerically. One of them is DIDO, commercially available from Tomlab Optimization. DIDO uses the pseudospectral approximation algorithm. (Ross 2004)

The OCP solver tool is supplied with a mathematical description of dynamic equations, all boundary conditions of states, all state and control limits and the optimization criterion. In DIDO all the descriptions are programmed as MATLAB-functions.

\section{Tractor-Trailer IN HEAdLANDS}

As stated previously the time spent in headlands is crucial when minimizing the time spent in the whole field. The turning of the tractor-trailer combination in headlands is studied below. The parameters used in this case are shown in table 1.

Table 1. The fixed parameters of the tractor-trailer combination.

\begin{tabular}{|cc|cc|}
\hline $\mathrm{a}(\mathrm{m})$ & 3 & $v_{R}^{\max }(\mathrm{km} / \mathrm{h})$ & 10 \\
\hline $\mathrm{b}(\mathrm{m})$ & 1 & $a_{R}^{\max }=-a_{R}^{\min }(\mathrm{km} / \mathrm{h} / \mathrm{s})$ & 2 \\
\hline $\mathrm{c}(\mathrm{m})$ & 4 & $\alpha_{F}^{\max }=-\alpha_{F}^{\min }\left({ }^{\circ}\right)$ & 45 \\
\hline $\mathrm{d}(\mathrm{m})$ & 1.5 & $\omega_{F}^{\max }=-\omega_{F}^{\min }(\% / \mathrm{s})$ & 40 \\
\hline$v_{R}^{\min }(\mathrm{km} / \mathrm{h})$ & -5 & $\varphi^{\max }=-\varphi^{\min }\left({ }^{\circ}\right)$ & 60 \\
\hline
\end{tabular}

The optimization criterion $J$ is minimum time or $F=1$.

The headland type here is selected so that the headlands boundary is straight and the width $\left(w_{h}\right)$ and the angle $\left(\beta_{h}\right)$ of headlands are variables. The situation in headlands is presented in figure 4 . 


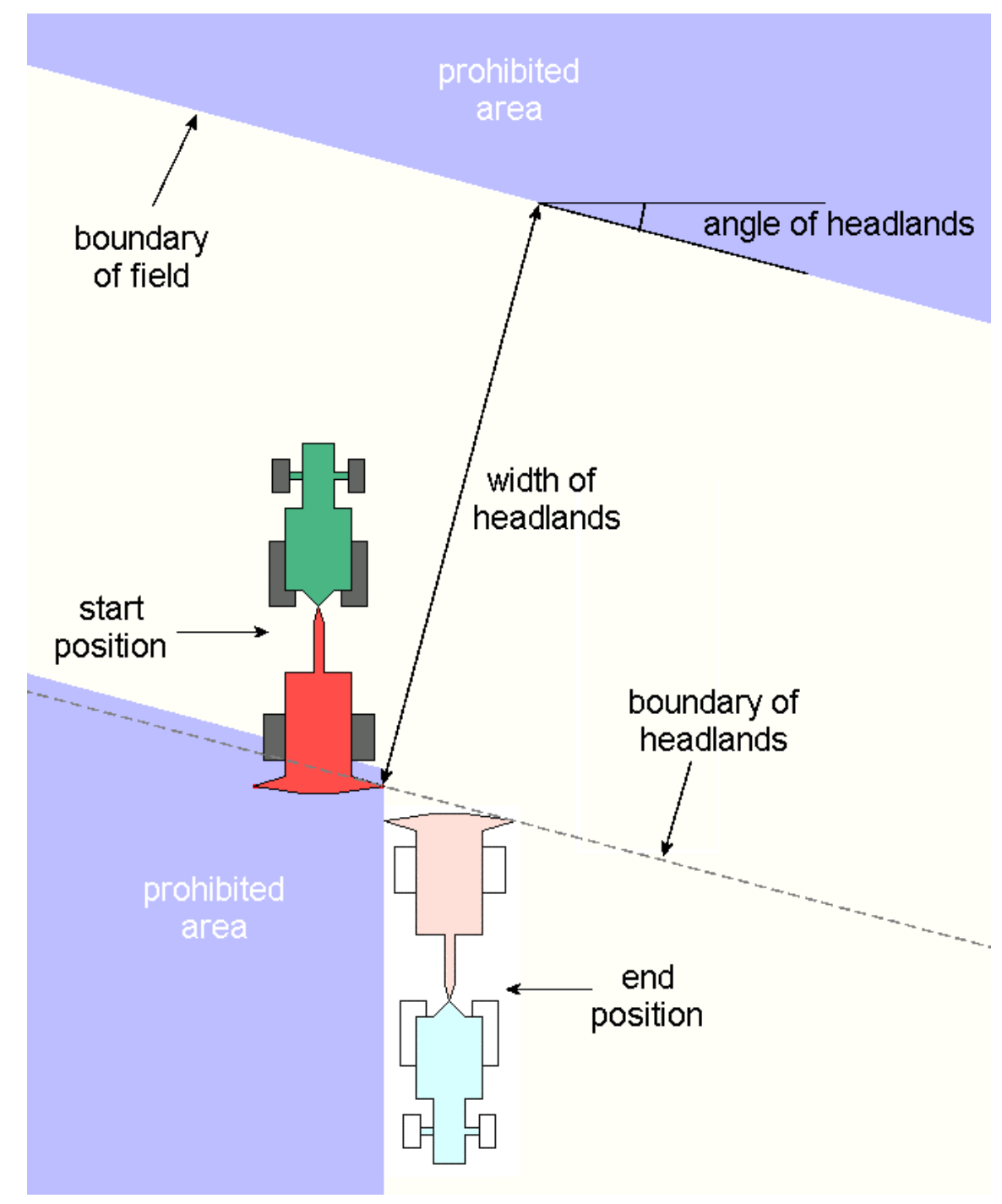

Figure 4. Headlands situation and parameters

The problem is to find the optimal trajectory (for states and controls) in order to move the vehicle from the start position to the end position. The problem is formulated as an OCP and solved with DIDO. A minor problem with solving is that it takes quite much time from a desktop computer, i.e. the solution of OCP with fixed headlands parameters takes usually more than five minutes. That is why the solutions (trajectories) have to be approximated in order to utilize the method in real-time applications.

One of the problems in solving the optimal control problem with a numerical algorithm is the periodicity of angle. The problem exists in the boundary conditions, where the end boundary condition differs by $2 \pi$ if the turning has been made using reverse-back turning compared with pure forward driving. In reverse-back turning the angle difference between start and end position angles is $\pi$ and in forward turning it is $-\pi$. This problem has been worked around by solving the OCP in both cases and selecting best of them.

The example solution for a right hand side turning is presented in figure 5 . The parameters in this example are width $=17 \mathrm{~m}$, angle $=-15^{\circ}$. Twenty discrete points in time are used. 


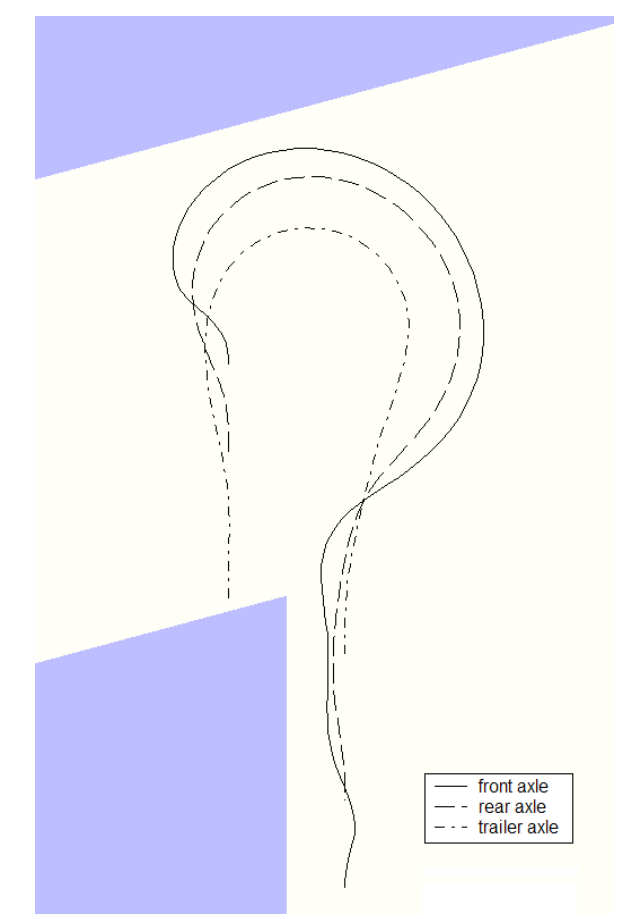

Figure 5. Example of optimal turning path, right hand side turning

\section{The EfFect Of ANGLe ANd WidTh OF Headlands}

Headland parameters tried were width from 14 to 20 meters and angle from -60 to $60^{\circ}$ with 7 and 8 samples, respectively. Both the left and right hand side turning were solved. The turning time in both turning cases with varying headland angle and width is presented in figure 6 . Vertical bars correspond to time. The left side bar in each position represents the time spent in turning where the nose of tractor has turned to left by $\pi\left(180^{\circ}\right)$, and right side bar oppositely. Longer one of the pair of bars in each position means longer turning time. The difference of left and right hand side turning time is presented also in figure 6 . If the horizontal bar below points to right, it means that right hand side turning is preferred, the longer the more preferred.

In figure 6 it can be also seen that both turning types are not always possible or far from time optimal. It can be said that with narrow headland width and small angle the right side solution is not possible. In some cases the numerical solution is not found at all. These results apply only to the vehicle used in this example, for other parameters the results will be different. 


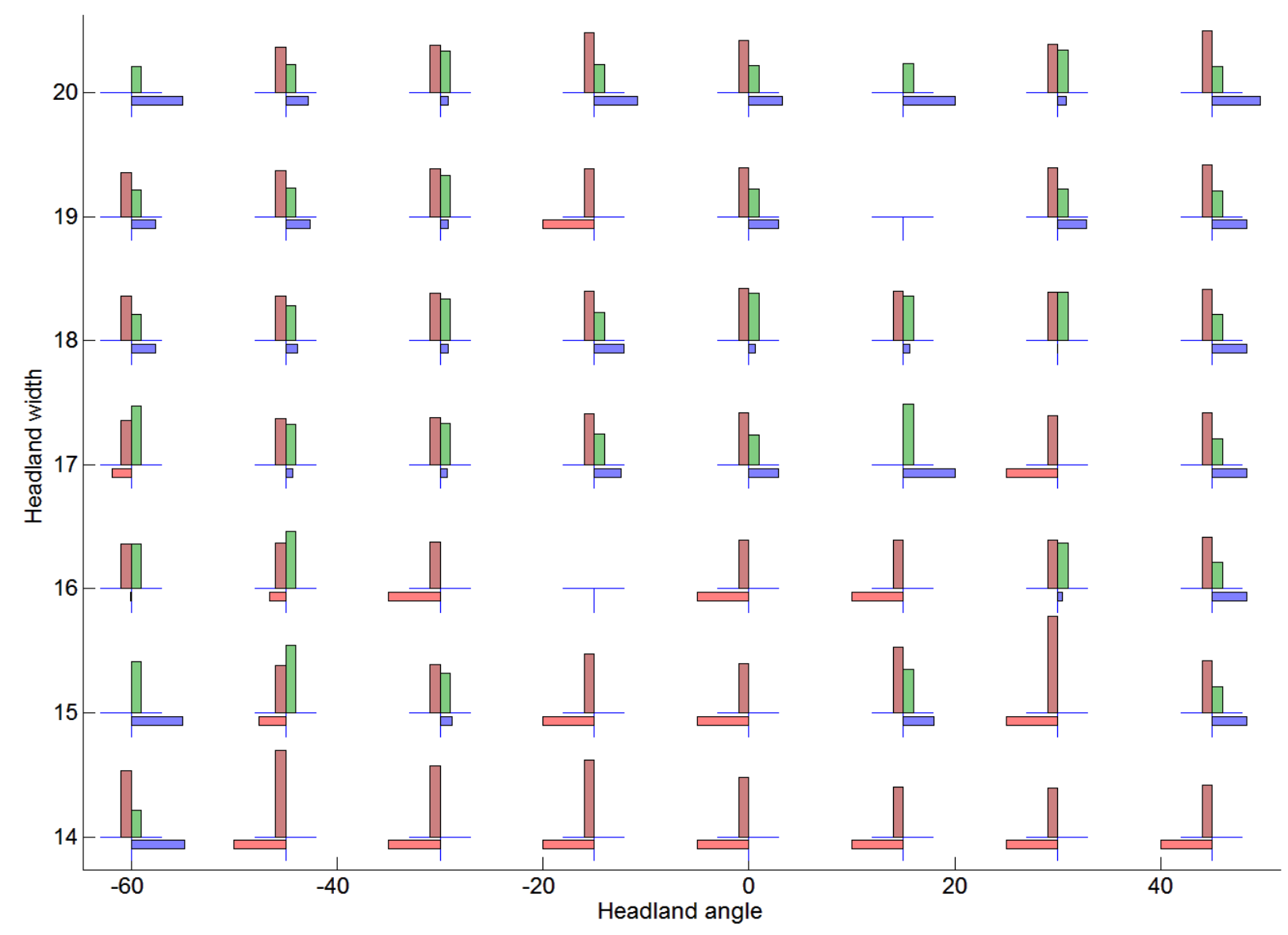

Figure 6. Turning time with varying headland angle and width.

The problems of a numerical OCP solver can be seen in the solutions. The solution found is usually a local, not global, optimum.

\section{APPRoximation OF PATH}

The solutions of the OCP are trajectories of states and controls. The controls and last two states (velocity and turning angle and time derivatives of them) can be omitted in the approximation because the controls can be derived from the states in real time trajectory following application. The vehicle control system can be built so that it can follow a certain 2D-path, so the reference path for vehicle is needed.

Numerical OCP solvers give the trajectories in a discretized form, but a continuous reference path is better for the controller. Piecewise Bezier curve method has been tried.

A Bezier curve is tuned with eight parameters, four of which fix both end points of the curve. The other four are used modifying the shape of the curve or shaping control points. Piecewise approximation is made so that first bezier curve is fitted to path data from start to such position where the approximation error is within certain limits. Here the suitable error is assumed so that maximum error in path is $5 \mathrm{~cm}$. Next bezier curve is fitted from the end of last bezier curve. Two consecutive curves have common parameters so that the end point $(\mathrm{x}, \mathrm{y})$ of last and start point of next are the same but so is the tangent, so there are five parameters to be fixed within one place. Cubic bezier curve formula is expressed in (9), A and D are the end points, B and C are the control points.

$$
P_{B}(w)=w^{3} A+3 w^{2}(1-w) B+3 w(1-w)^{2} C+(1-w)^{3} D, w \in[0,1]
$$

Piecewise bezier curve approximation is presented in figure 7. The 2D-path of tractor's rear axle center point is used. The bold line is the approximation and the continuous line the original in discrete points $\mathrm{x}$. 


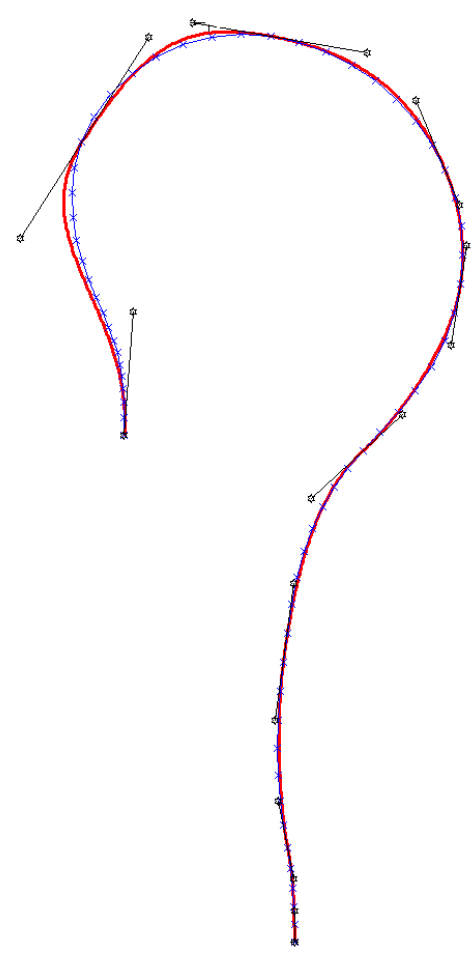

Figure 7. Piecewise bezier curve approximation of the path.

The result is quite good, the only problem is that bezier curve cannot approximate well circles which can be seen also from figure 7 .

\section{CONCLUSION}

Automatic path creating in headlands is important if the field vehicles are fully automatized. The path of a tractor-trailer combination in specified headland can be solved using an optimal control solver. Numerical optimal control problem solvers still have some drawbacks, most important of which are the uncertainty of solution convergence and the numerical calculation power needed from the computer. The path can be approximated with bezier curves.

Next the reference path creation between parameter combinations should be tried without using an OCP solver, but by generating from nearby parameter combination solutions. Other methods for continuous path approximation should be tried in order to get smoother reference path with less parameters.

\section{REFERENCES}

1. von Stryk, O., and Bulirsch, R. 1992. Direct and indirect methods for trajectory optimization. Annals of Operation Research. 37(1992): 357-373.

2. Ross, I.M., and Fahroo, F. 2002. A direct method for solving nonsmooth optimal control problems. Proceedings of the 2002 IFAC World Congress, Barcelona, Spain. July 2002.

3. Ross, I.M. 2004. User manual for DIDO.

4. Bryson Jr. A. E. 1996. Optimal control 1950 to 1985. IEEE Control Systems, 16(3):26-33. 